\title{
Depresión y prácticas religiosas en internos de un establecimiento penitenciario de Cusco-Perú
}

\author{
Viki Yunguri Arias ${ }^{1}$, Elizabeth Gutiérrez Suna ${ }^{2}$, Vilma Aurora Pacheco \\ Sota ${ }^{3}$, Yeny Maritza Accostupa Quispe ${ }^{4}$, Cleto De La Torre Dueñas ${ }^{5}$, \\ Tesania Velázquez ${ }^{6}$ \\ Universidad Nacional de San Antonio Abad del Cusco ${ }^{1,2,3,4,5}$, Pontificia \\ Universidad Católica del Perú ${ }^{6}$
}

\begin{abstract}
El objetivo del estudio fue determinar la relación entre depresión y prácticas religiosas en internos de un Establecimiento Penitenciario de Cusco-Perú. Se aplicó, el Inventario de Depresión de Beck (BDI - II) (Beck et al., 2006) y la Escala de Prácticas Religiosas adaptada al contexto penitenciario (Hamann, 2003) a una muestra de 380 internos por distintos delitos. Los resultados indican que no hay una relación entre depresión y prácticas religiosas; sin embargo, se encontró relación con el indicador "Me comporto como Dios espera que lo haga". También se obtuvo que el $71 \%$ de la muestra tiene algún nivel de depresión. De estos, $24.7 \%$ de internos tiene depresión leve, $22.45 \%$ depresión moderada y $24.2 \%$ depresión grave. Asimismo, se obtuvo que el $62.8 \%$ de internos tienen prácticas religiosas, las más frecuentes son: $61.1 \%$ reza a Dios para pedirle ayuda, $56.1 \%$ agradece a Dios, $36.6 \%$ tras-
\end{abstract}

1 Licenciada en Psicología de la Universidad Nacional de San Antonio Abad del Cusco. Secretaria Técnica del Centro de Investigación y Encuestas CIEN-PERÚ. Dirección postal: Av. La Cultura n733, Cusco 921, Perú. Correo electrónico: a20146548@pucp.pe

2 Licenciada en Psicología de la Universidad Nacional de San Antonio Abad del Cusco. Dirección postal: Av. La Cultura n³3, Cusco 921, Perú. Correo electrónico: elita5784@ hotmail.com

3 Magíster en Psicología Educativa. Directora de la Dirección de Registro y Servicios Académicos y docente de la Universidad Nacional de San Antonio Abad del Cusco. Dirección postal: Av. La Cultura n733, Cusco 921, Perú. Correo electrónico: vimaury69@hotmail.com

4 Magíster en Física Aplicada por la Pontificia Universidad Católica del Perú y docente de la Universidad Nacional de San Antonio Abad del Cusco. Dirección postal: Av. La Cultura $n^{\circ} 733$, Cusco 921, Perú. Correo electrónico: yeny.accostupa@unsaac.edu.pe

5 Magíster en Estadística por la Universidad Nacional Mayor de San Marcos. Director de la Unidad de Postgrado de la Facultad de Ciencias y docente de la Universidad Nacional de San Antonio Abad del Cusco. Dirección postal: Av. La Cultura n733, Cusco 921, Perú. Correo electrónico: Cleto.delatorre@unsaac.edu.pe

6 Magíster en Psicología Clínica y Forense por la Universidad de Salamanca. Directora de la Dirección Académica de Responsabilidad Social y docente del Departamento de Psicología y de la Maestría en Psicología Comunitaria de la Pontificia Universidad Católica del Perú. Coordinadora de grupo de investigación de psicología forense y penitenciaria de la PUCP. Dirección postal: Av. Universitaria 1801, San Miguel, Lima 32, Perú. Correo electrónico: tvelazq@pucp.pe 
mite su fe a personas cercanas, $25.5 \%$ lee la biblia y $25 \%$ asiste a celebraciones religiosas realizadas dentro del Penal.

Palabras clave: Depresión, prácticas religiosas, internos, establecimiento penitenciario.

\section{Depression and religious practices in inmates of a prison in Cusco-Peru}

The objective of the study was to determine the relationship between depression and religious practices in inmates of a Penitentiary Establishment in Cusco-Peru. The Beck Depression Inventory (BDI - II) (Beck et al., 2006) and the Religious Practices Scale adapted to the prison context (Hamann, 2003) were applied to a sample of 380 inmates for different crimes. The results show that there is no relationship between depression and religious practices. However, a relationship was found with the indicator "I behave as God expects me to do it". $71 \%$ of the sample has some level of depression: $24.7 \%$ of inmates have mild depression, $22.45 \%$ moderate depression and $24.2 \%$ severe depression. It was also obtained that $62.8 \%$ of inmates have religious practices. The most frequent are pray to God to ask for help $(61.1 \%)$, thank God $(56.1 \%)$, transmit their faith to people close to them $(36.6 \%)$, read the Bible (25.5\%) and attend religious celebrations held inside the prison (25\%).

Keywords: Depression, religious practices, internal, penitentiary establishment.

\section{Depressão e práticas religiosas em presidiários de uma prisão em Cusco-Peru}

O objetivo do estudo foi determinar a relaçáo entre depressão e práticas religiosas em presidiários de um estabelecimento penitenciário de Cusco-Peru. Foi aplicado, o inventário de depressão de Beck (BDI - II) (Beck et al., 2006) e religiosos Práticas de escala adaptada ao contexto penitenciária (Hamann, 2003) para uma amostra de 380 presos para vários crimes. Os resultados indicam que não há relação entre depressão e práticas religiosas, entretanto, foi encontrada uma relaçáo com o indicador "Eu me comporto como Deus espera que eu faça", também foi obtido que $71 \%$ da amostra tem algum nível de depressão destes $24.7 \%$ dos internos têm depressão leve, $22.45 \%$ depressão moderada e $24.2 \%$ depressão grave. Também foi obtido que $62.8 \%$ dos presos possuem práticas religiosas, sendo as mais frequentes; $61.1 \%$ reza a Deus por ajuda, graças a Deus $56.1 \%, 36.6 \%$ transmitida às pessoas fechar sua fé, $25.5 \%$ lêem a Bíblia e $25 \%$ frequentam celebraçôes religiosas realizadas dentro da prisão.

Palavras-chave: Depressão, práticas religiosas, interno, estabelecimento penitenciário.

\section{Dépression et pratiques religieuses chez les détenus d'une prison de Cusco-Pérou}

L'objectif de l'étude était de déterminer la relation entre la dépression et les pratiques religieuses chez les détenus d'un établissement pénitentiaire de Cusco-Pérou. Le Beck Depression Inventory (BDI-II) (Beck et al., 2006) et l'échelle des pratiques religieuses adaptées au contexte carcéral (Hamann, 2003) ont été appliqués à un échantillon de 380 détenus pour différents crimes. Les résultats indiquent qu'il n'y a pas de relation entre la dépression et les pratiques religieuses; cependant, une relation a été trouvée avec l'indicateur "Je me comporte comme Dieu l'attend». Il a également été constaté que 71\% de l'échantillon a un certain niveau de dépression. Parmi ceux-ci, $24.7 \%$ des détenus souffrent de dépression légère, $22.45 \%$ de dépression modérée et $24.2 \%$ de dépression sévère. De même, il a été obtenu que $62.8 \%$ des détenus ont des pratiques religieuses, les plus fréquentes sont: $61.1 \%$ prient Dieu pour l'aide, $56.1 \%$ remercient Dieu, 36.6\% transmettent leur foi aux proches, $25.5 \%$ lisent la Bible et $25 \%$ assistent aux célébrations religieuses organisées dans la prison. Mots clés: Dépression, pratiques religieuses, détenus, établissement pénitentiaire. 


\section{Introducción}

La Organización Mundial de la Salud (2017) indica que la depresión, como enfermedad mental, afecta a 350 millones de individuos, mientras que en las Américas cerca de 50 millones de personas la padecieron en el año 2015. Así, el encarcelamiento no solo implica la pérdida de la libertad, sino que implica ingresar a un nuevo entorno al cual es necesario adaptarse, en este entorno, la persona encarcelada pierde contacto con su familia, amigos, trabajo, afectándose así sus relaciones sociales, laborales y sexuales (Mikulic \& Crespi, 2005; Ruiz, 2007; Valverde, 1991). En los establecimientos penitenciarios la salud mental es un tema de investigación reciente. Las personas recluidas en estos centros no son ajenas a este problema de salud pública por su elevada prevalencia, impacto en la calidad de vida, la marcada discapacidad que produce y el incremento en el uso de los servicios de salud (Mori \& Caballero, 2010).

Más aún cuando en los últimos 15 años la población penitenciaria se ha incrementado de manera considerable en América Latina. Así, en países como Brasil, Colombia y México ha aumentado en 150\%, 125\% y 53\%, respectivamente (Penal Reform International, 2015). El Perú no es la excepción, habiéndose duplicado la población penitenciaria en los últimos años.

Según refiere el Instituto Nacional Penitenciario (INPE, 2017), en el Informe Estadístico la población del sistema penitenciario al mes de setiembre 2017 es de 102,865 personas, el incremento ha sido de $6 \%$ (4,530 internos) en un lapso de doce meses. En cuanto a la salud mental, el encarcelamiento genera por sobre todo depresión y ansiedad, además de incrementar los problemas conductuales de las personas recluidas desencadenándose problemas en la convivencia (Borja et al., 2006). 
Asimismo, Valverde (1991) indica en primer lugar, el ambiente dentro de un establecimiento penitenciario es visto por las personas encarceladas como peligroso, por lo que se sienten vulnerables a cualquier acto de violencia que pudiese ocurrir en el interior, en segundo lugar, la infraestructura rígida y la violencia lleva a que se genere agentes de control social informales, por ello, los internos generan códigos y señales propios de comunicación.

El encarcelamiento genera cambios en las personas. Valverde (1991) menciona cambios relacionados al cuerpo, cambios psicosociales y cambios asociados a la adaptación al entorno. Entre los cambios asociados al cuerpo, encuentra problemas sensoriales y agarrotamiento de los músculos. Entre los cambios psicosociales, describe la ausencia de control sobre la propia vida, el estado permanente de ansiedad, la ausencia de expectativas a futuro, la ausencia del sentimiento de responsabilidad frente a los propios actos, la pérdida de vinculaciones, alteraciones en el uso del lenguaje, y alteraciones de la afectividad como sensación de desamparo y sobredemanda afectiva. Menciona, también, cuatro cambios asociados a la adaptación al entorno anormal de la prisión: exageración de las situaciones, autoafirmación agresiva o sumisión frente a la institución, dominio o sumisión en las relaciones interpersonales y alteración de la sexualidad.

Sumado a ello, Castro (2009), refiere que "La prisionalización afecta en la personalidad del recluso y la altera durante el internamiento, dejando secuelas psíquicas leves o profundas, a veces irreversibles, otras dificultades, lamentables, de los penales del Perú, son: la sobrepoblación penitenciaria; la inseguridad de sus instalaciones o los problemas de alimentación; los presos sin condena y un sin fin de privaciones que tienen que soportar los internos" (p.113). En este panorama, los trastornos depresivos son un problema importante en la salud mental de las personas en centros de reclusión (Dasso, 2010).

Así, según el Centro Nacional de Estudios Criminológicos y Penitenciarios (CENECP, 2007) el 15.71\% de la población penitenciaria peruana sufre de algún trastorno depresivo y casi el 70\% de todos los internos posee algún tipo de sintomatología depresiva. Asimismo, 
según el Instituto Nacional de Estadística e Informática (INEI, 2016), en su Primer Censo Nacional Penitenciario encontró que el tipo de enfermedad que ocupa el primer lugar es la depresión con un $9.6 \%$ de la población penal, es decir, 7 mil 321 internos(as), seguido por el $8.7 \%$ de internos(as) con ansiedad, el 8.4\% con enfermedad pulmonar crónica y el $7.0 \%$ con hipertensión, entre otros.

Mojica et al., (2009), hallaron que el 20\% de los internos de los centros penitenciarios tienen riesgo suicida, así como, manifestación de desesperanza y depresión. En otro caso, Ordóñez y Ruiz (2008), revisaron estadísticas que aseguran mayor prevalencia de suicidios en prisión que en población civil externa. La Asociación Psiquiátrica Peruana (APP, 2011), señala que por lo menos seis de cada diez internos presentan cuadros psicóticos que los hacen caer en depresión, los vuelven agresivos e incluso los hacen concebir ideas autodestructivas.

Abonando, la Defensoría del Pueblo (2011) encontró que en los penales del país habían 34 personas privadas de libertad declaradas "inimputables" por el Poder Judicial, pero que simplemente no abandonaban la cárcel porque no eran recibidos en hospitales psiquiátricos. Además, 145 internos eran considerados pacientes psiquiátricos, es decir, aquellos que necesitaban un tratamiento y en muchos casos una medicación, pero señalaban que las cifras pueden no reflejar con certeza la realidad debido a las limitaciones existentes en el registro de casos por parte del INPE.

Los datos más recientes de la Defensoría del Pueblo (2017) indican que por las visitas realizadas a cada establecimiento penitenciario el principal problema es el hacinamiento. La población penitenciaria era de 2611 internos para una capacidad de albergue de 1200. Asimismo, los internos indicaron que muchos habían sido trasladados hace tres años de manera indefinida del penal a causa del hacinamiento. En la visita a los establecimientos penitenciarios se evidenció diversos problemas como la ausencia de ambientes adecuados, así como dificultades en la atención de salud, se encontró que las condiciones del ambiente de meditación no cumplían con los requisitos mínimos de habitabilidad ni salubridad, los establecimientos penitenciarios no cuentan con 
profesionales de tratamiento como asistencia social, lo que limita el tratamiento penitenciario básico.

Durante la supervisión hallaron una situación muy grave: de los 122 internos con TBC, 108 tenían como diagnóstico la multirresistencia a los fármacos (MDR), y tres de ellos estaban diagnosticados con $\mathrm{XMDR}$, es decir, con muy alta resistencia al tratamiento, agravándose aún más su salud y aumentando la posibilidad de contagio. El mes de marzo se visitó los penales de Arequipa (hombres y mujeres). Uno de los principales problemas es que desde el 2016 no se había dado mantenimiento a la máquina que trata el agua empleada dentro del penal ni tenía la certificación de agua apta para el consumo humano otorgado por el Minsa. En el mes de mayo visitaron los establecimientos penitenciarios de Tarapoto, Moyobamba y Pampas de Sananguillo, ubicados en San Martín. En todos, la mayor problemática es la asignación de un solo profesional en el órgano técnico de tratamiento para una población de más de 500 internos y la ausencia de un médico por penal; en la mayoría de los casos, un técnico en enfermería cumple esta labor. En setiembre se supervisaron los penales de Huancayo, Concepción, Jauja, Iquitos, Trujillo, Challapalca y Puno, verificándose sobrepoblación (con excepción de Challapalca), falta de personal de salud, carencia de profesionales de tratamiento, entre otros (Defensoría del Pueblo, 2017).

En octubre se supervisaron los penales de Chiclayo y Pucallpa, y en noviembre los penales de Cusco, Ica y Chincha. Los problemas comunes están vinculados a deficientes condiciones carcelarias, carencia de servicios de salud y limitación de personal en el tratamiento penitenciario (Defensoría del Pueblo, 2017).

Por las visitas realizadas a los establecimientos penitenciarios indican que las condiciones de las personas privadas de libertad no son óptimas (Defensoría del Pueblo, 2017) todas estas condiciones hacen que el interno desarrolle sintomatología depresiva, siendo la depresión un problema de salud público en estos ambientes. Es así, que el tema de depresión en internos en los establecimientos penitenciarios ha sido poco estudiado. Esto es causado, según Palmer \& Connelly (2005), 
porque los internos con depresión no suelen estar dispuestos a colaborar con las investigaciones; se sabe que los encarcelamientos prolongados comprometen la salud mental de los internos, teniendo manifestaciones de la sintomatología depresiva como: pérdida del interés y del placer de actividades normales, fatiga, aislamiento, aumento o disminución involuntario de peso, apatía, insomnio o aumento del sueńo, animo depresivo, pesimismo, minusvalía, desesperanza e intento de suicidio.

Las prácticas religiosas se abordan en su implicancia emocional y cognitiva antes que en su significado ontológico porque no se pretende discutir la existencia de Dios, sino el valor o repercusión de las prácticas religiosas en la salud y el equilibrio del interno. Se ha procurado un acercamiento desde el punto de vista funcional y sus impactos sobre lo individual al preguntar si existe o no relación entre la depresión y las prácticas religiosas, y si es posible que las prácticas religiosas, a través de ciertos actos especiales como: ritos o rituales religiosos -a los que en sentido restringido a veces también se los denomina culto- ayudan en la disminución de la depresión.

Esta pregunta parte de la constatación que, en la población estudiada, las prácticas religiosas son muy arraigadas, ya que la mayoría de los internos profesan y se encuentran afiliados a una religión dentro del penal. Así, según el INEI (2016), los privados de libertad profesan en mayor porcentaje la religión católica (63.5\%), evangélica (25\%) y adventista (1.6\%). En cada religión realizan prácticas religiosas a nivel personal y grupal.

Además, esta pregunta se asienta en que los agentes pastorales como organizaciones que trabajan en coordinación con el INPE buscan brindar asistencia religiosa y ayudar en la rehabilitación de la persona privada de su libertad (INPE, 2018). Es más, teóricamente la religiosidad podría ligarse a una mejora en la persona, sintiéndose más fuerte si es que esta se encuentra ante alguna adversidad tanto a nivel psicológico como físico (Acosta y Sánchez, 2017). Porque las creencias religiosas contribuyen a la búsqueda de un balance personal, además de dar mejores condiciones a los individuos para hacer frente a la adversidad (Chaves et al., 2014). 
Finalmente, la religión inspira en la mayoría de las personas fortaleza y valor, porque proporciona elementos para que se enfrente la situación de encarcelamiento (Ocampo et al., 2006).

De todo lo expuesto podría inferirse que la privación de la libertad es un evento detonador de la depresión, mientras que factores como las prácticas religiosas podrían estar actuando como factor protector, en tanto fortalece la resiliencia de los internos. De aquí el interés por determinar si realmente existe o no relación entre ambas variables.

Respecto al ambiente de estudio, el Establecimiento Penitenciario fue construido en el primer gobierno del presidente Fernando Belaunde Terry en 1951 y abierto por Resolución Suprema No 211 del 13 de mayo de 1965 (Instituto Nacional Penitenciario [INPE], 2011).

Desde el momento en que la persona ingresa al Establecimiento Penitenciario se le denomina interno, teniendo una situación jurídica de procesado o sentenciado. Durante el primer mes el interno permanece en un ambiente de aislamiento, luego el Órgano Técnico de Tratamiento (OTT) del Establecimiento Penitenciario, organiza una Junta Técnica de Clasificación con los profesionales de asistencia; psicológica, social y legal, donde se evalúa y determina el régimen, nivel de peligrosidad y pabellón que corresponde al interno. Para esta clasificación se utiliza fichas conforme a la siguiente equivalencia, mínima peligrosidad igual mínima seguridad, mediana peligrosidad igual a mediana seguridad, máxima peligrosidad igual a máxima seguridad y difícil readaptación igual a Régimen Cerrado Especial. En el caso del Establecimiento Penitenciario no se cuenta con el Régimen Cerrado Especial debido a la sobrepoblación, hacinamiento y por seguridad del Penal por ello si el interno llega a este régimen es trasladado a otro penal de mayor seguridad. Esta clasificación se basa en variables e indicadores, como: número de ingresos, edad, número de agentes que participaron en el delito, modalidad del delito atribuido, penalidad al delito atribuido, signos relacionados a la conducta delictiva, responsabilidad frente al delito, adaptación a las normas y valores sociales, historia psicocriminológica, evaluación de la conducta delictiva, dinámica familiar, nivel de instrucción, comportamiento laboral y/o educativo, grupo social de referencia y repercusión social. 
Existen 5 pabellones divididos por cuadras, en estas cuadras los espacios de los dormitorios son muy reducidos y se encuentran tan hacinados que cada interno, por sus medios, tiene que comprar o buscar un espacio para dormir lo que suma a las dificultades para conciliar el sueño generado por sus preocupaciones legales, familiares, económicas o a las constantes pesadillas.

Para realizar el aseo personal tienen que madrugar antes de las 5 de la mańana porque pasada esa hora ya no abastece el agua para tantos internos, lo cual genera malestar. Los servicio de salud que brinda el Establecimiento Penitenciario de medicina general y odontología son muy limitados, ya que para una población penal de 2680 internos aproximadamente, solo se cuenta con dos profesionales de salud, además de no tener la implementación adecuada, lo que deviene en una preocupación constante respecto a su salud. Las áreas de trabajo se encuentran limitadas por el hacimiento de internos, por ello el interno opta por trabajar en los patios, canchas deportivas y pasadizos.

En cuanto a las visitas, al inicio de su encarcelamiento son muy frecuentes, pero según pasa el tiempo las visitas van disminuyendo, hasta desaparecer. Las visitas refieren que son maltratadas a la hora de hacer la cola y pasar revisión en cada puerta de ingreso, lo que se constituye en otra fuente de preocupación para el interno y sus familiares.

La atención psicológica, social y legal no responde a la demanda de la población penal, siendo esto un factor en perjuicio para la evaluación del interno, no se cuenta con el número de profesionales necesarios ni con los recursos adecuados para dar cuenta de la progresión en la conducta del interno.

Todo lo descrito anteriormente hace que el interno experimente tristeza, pesimismo, fracaso por encontrarse encerrado y no poder ayudar a su familia e hijos, pérdida de placer para realizar cualquier actividad, sentimientos de culpa por el delito cometido, disconformidad consigo mismo, autocritica, pensamientos o deseos suicidas, llanto, agitación, pérdida de interés, indecisión, desvalorización, pérdida de energía, cambios en los hábitos de sueńo, irritabilidad, cambios de apetito, dificultad de concentración en las actividades que realizan 
dentro del Establecimiento Penitenciario, cansancio o fatiga, pérdida o aumento de interés por el sexo (actividad sexual).

Por todo lo mencionado anteriormente en muchos casos el interno busca un refugio en Dios (o en prácticas religiosas), mediante el rezo o las oraciones, asistencia a misa o culto, durante estas celebraciones realizan cantos, alabanzas a Dios donde piden perdón de rodillas y lloran por el delito que cometieron. También la mayoría de los internos cuentan con una biblia para leer la palabra de Dios y hallar consuelo en ella, algunos internos se dedican a predicar la palabra de Dios a sus compańeros formando pequeños grupos; los movimientos religiosos y el área de asistencia social realizan el sacramento de bautizo para aquellos internos que no fueron bautizados, también realizan la primera comunión, confirmación y matrimonio en coordinación con los directivos del Establecimiento Penitenciario.

El Establecimiento Penitenciario cuenta con varias capillas. Cada pabellón cuenta con una pequeńa capilla, las capillas generales y existen imágenes de santos católicos como Patrón San Jerónimo, Señor de Qoyllorit'i, Señor de Huanca, Virgen María, Santa Rosa, Sarita Colonia, Virgen de Guadalupe, entre otras. Se ha encontrado la presencia de diferentes grupos religiosos que realizan asistencia espiritual a los internos, como La Vid, Movimiento Misionero Mundial (MMM), iglesia Católica, los cuales realizan su labor los fines de semana, así como el Ministerio Público por medio de su programa Kusikuy. Por otro lado, el Establecimiento Penitenciario de Cusco organiza celebraciones religiosas como misas para toda la población penal en su conjunto.

En este contexto la religiosidad podría ser una alternativa para hacer frente a los problemas del estado de ánimo presentes entre los internos. Entonces, para esta investigación se trabajó con las siguientes interrogantes ¿Existe relación entre depresión y prácticas religiosas?, ¿Cuál es el nivel de depresión?, ¿Cuáles son las prácticas religiosas más comunes? y ¿Existe relación entre los indicadores de la Escala de Prácticas Religiosas con la depresión en los internos del Establecimiento Penitenciario Penal de Cusco? 
Depresión y prácticas religiosas en internos de un establecimiento penitenciario / Yunguri et al.

\section{Método}

\section{Participantes}

La muestra de estudio estuvo conformada por 380 internos de un Establecimiento Penitenciario de Cusco-Perú. Los participantes son internos mayores de 18 años recluidos por distintos delitos como Tráfico ilícito de drogas, Robo agravado, Hurto, Violación sexual, Secuestro, Estafa, Actos contra el pudor, Homicidio, Lesiones graves y Omisión familiar, con condición jurídica de procesado o sentenciado. En la tabla 1 aparecen las características sociodemográficas de los participantes.

\section{Tabla 1}

Características sociodemográficas de internos del Establecimiento Penitenciario de Cusco

\begin{tabular}{lcc}
\hline Características sociodemográficas & $f$ & $\%$ \\
\hline Rango de edades & 61 & 16.1 \\
18 - 24 años & 76 & 20 \\
25 -29 años & 200 & 52.6 \\
30 - 44 años & 42 & 11.1 \\
45 - 59 años & 1 & 0.3 \\
60 a mas & 43 & \\
\hline Grado de instrucción & 34 & 11.3 \\
Primaria incompleta & 98 & 8.9 \\
Primaria completa & 138 & 25.8 \\
Secundaria incompleta & 44 & 36.6 \\
Secundaria completa & 7 & 11.6 \\
Superior no universitaria incompleta & 16 & 1.8 \\
Superior no universitaria completa & 4.2 \\
Superior universitaria incompleta & & \\
\hline
\end{tabular}




\begin{tabular}{lcc}
\hline Características sociodemográficas & $f$ & $\%$ \\
\hline Situación jurídica & 32 & 8.4 \\
Procesado & 348 & 91.6 \\
Sentenciado & & \\
\hline Tiempo de reclusión & 36 & 9.5 \\
Menos de un año & 200 & 52.6 \\
De 1 a 5 años & 96 & 25.3 \\
De 5 a 10 años & 41 & 10.8 \\
De 10 a 15 años & 7 & 1.8 \\
De 15 a más & & \\
\hline Religión & 254 & 66.8 \\
Católica & 100 & 26.3 \\
Evangélica & 8 & 2.1 \\
Adventista & 7 & 1.8 \\
Testigo de Jehová & 11 & 2.9 \\
Ninguna & & \\
\hline
\end{tabular}
$n=380$

En la tabla 1, se observa que el rango de edad de los participantes estuvo entre 18 a más de 60 años, el grupo de mayor población es entre 30-44 ańos (52.6\%). Por otra parte, el grado de instrucción va de primaria incompleta hasta superior universitaria incompleta, siendo el nivel educativo, mayoritariamente alcanzado el de secundaria completa (36.6\%). Según la situación jurídica el $8.4 \%$ es procesado y el $91.6 \%$ sentenciado. El tiempo de reclusión del $52.6 \%$ se encuentra entre uno y cinco años; y, con respecto a la religión el $66.8 \%$ es católico y el $26.3 \%$ evangélico.

\section{Medición}

Para el estudio se empleó una ficha sociodemográfica y dos instrumentos psicológicos. La ficha sociodemográfica fue aplicada para 
conocer los datos básicos de los participantes como: edad, grado de instrucción, situación jurídica, tiempo de reclusión, religión y otros datos vinculados con el estudio.

Los instrumentos aplicados fueron el Inventario de Depresión de Beck (BDI - II) (Beck et. al, 2006), para evaluar la depresión y la Escala de Prácticas Religiosas adaptada al contexto penitenciario (Hamann, 2003), para evaluar las prácticas religiosas. El Inventario de Depresión de Beck (BDI - II), es un instrumento que mide la severidad de depresión en niveles como: mínima, leve, moderada y grave, consta de 21 ítems, los síntomas que mide son: tristeza, pesimismo, pérdida del placer, disconformidad con uno mismo, irritabilidad, dificultad de concentración, fatiga y pérdida en el interés en el sexo (Beck et al., 2006).

La Escala de Prácticas Religiosas adaptada al contexto penitenciario (Hamann, 2003), es una escala que evalúa las prácticas religiosas dentro del contexto penitenciario en niveles como; baja, media y alta, asimismo consta de 7 ítems.

Ambas pruebas fueron sometidos a análisis estadístico de confiabilidad en la población investigada, obteniéndose un alfa de Cronbach de .81 y .84 , respectivamente.

La baremación de los instrumentos fue utilizando como base las puntuaciones de escala Likert de cada instrumento, para obtener las puntuaciones de la variable de estudio, se sumaron los valores obtenidos respecto a cada ítem y para facilitar la interpretación se trasformó a una escala cualitativa mediante percentiles para ambos instrumentos, por tanto, en la baremación del Inventario de depresión de Beck, se trabajó con 3 puntos de corte, los cuales se dividen en percentiles que permiten evaluar el nivel de depresión como; mínima , leve , moderada o grave . La decisión de usar estos (u otros) puntos de corte se debe basar en las características concretas de la muestra y el propósito para el que se esté usando la prueba. Con respecto a la baremación de la Escala de Prácticas Religiosas se trabajó con dos puntos de corte, los cuales se dividen en percentiles, donde estos, permiten calificar el nivel de prácticas religiosas como; baja, mediana y alta . Se hizo el proceso de análisis y conversión de los datos en setiembre del 2018, una vez que 
se contó con toda la información pertinente, se realizó la baremación debido a que los instrumentos aplicados no se encontraban validados en población penitenciaria cusqueña.

\section{Procedimiento}

Se aplicó de manera conjunta con los cuestionarios una ficha sociodemográfica con información general sobre datos familiares y sociales del interno, así como sobre su situación jurídica.

La aplicación de los cuestionarios fue de forma individual, siguiendo las instrucciones recomendadas por los respectivos autores. Los aspectos éticos estuvieron presentes a lo largo del estudio, y se plasmaron en la explicación y aplicación del consentimiento informado a cada participante. Se les indicó además que podían retirarse del estudio si así lo desearan y que esta investigación no guardaba relación alguna con el área de tratamiento del Establecimiento Penitenciario.

\section{Análisis de Datos}

Para el análisis y tratamiento estadístico de los datos se usó el paquete estadístico SPSS (Statistical Package for the Social Sciences) versión 23. Se aplicó la prueba estadística de la Chi-Cuadrada $\left(\chi^{2}\right)$ para saber el grado de relación de ambas variables, asimismo se empleó estadística inferencial no paramétrica como el coeficiente de Tau - b de Kendall, ya que la distribución de los datos no eran normales. El estadístico que se utilizó para verificar la normalidad de la distribución fue la Prueba de normalidad de Kolmogorov-Smirnov (prueba K-S).

\section{Resultados}

En la tabla 2, se observa, la relación entre depresión y prácticas religiosas. Se encontró que la dimensión cognitiva de la depresión no presenta relación con la dimensión personal e interpersonal de las prácticas religiosas $(p=.954)$. Por otro lado, las dimensiones tanto 
conductual $(p=.712)$, y afectiva $(p=.230)$ no guardan relación con las dimensiones tanto personal como interpersonal de la depresión.

\section{Tabla 2}

Relación entre depresión y práctica religiosa

\begin{tabular}{|c|c|c|c|c|c|c|}
\hline & & & \multicolumn{3}{|c|}{ Práctica religiosa } & \multirow{2}{*}{ Total } \\
\hline & & & Alto & Medio & Bajo & \\
\hline \multirow{8}{*}{ Depresión } & \multirow[t]{2}{*}{ Mínima } & $f$ & 41 & 35 & 33 & 109 \\
\hline & & $\%$ & $37.6 \%$ & $32.1 \%$ & $30.3 \%$ & $100 \%$ \\
\hline & \multirow[t]{2}{*}{ Leve } & $f$ & 29 & 27 & 38 & 94 \\
\hline & & $\%$ & $30.9 \%$ & $28.7 \%$ & $40.4 \%$ & $100 \%$ \\
\hline & \multirow[t]{2}{*}{ Moderada } & $f$ & 31 & 29 & 25 & 85 \\
\hline & & $\%$ & $36.5 \%$ & $34.1 \%$ & $29.4 \%$ & $100 \%$ \\
\hline & \multirow[t]{2}{*}{ Grave } & $f$ & 40 & 19 & 33 & 92 \\
\hline & & $\%$ & $43.5 \%$ & $20.7 \%$ & $35.9 \%$ & $100 \%$ \\
\hline \multirow[t]{2}{*}{ Total } & & $f$ & 141 & 110 & 129 & 380 \\
\hline & & $\%$ & $37.1 \%$ & $28.9 \%$ & $33.9 \%$ & $100 \%$ \\
\hline
\end{tabular}

Se concluye que no hay relación entre la depresión y las prácticas religiosas en los internos del Establecimiento Penitenciario de Cusco. Los altos índices de depresión en los internos se explica por la falta de soporte familiar, ya que el $38.4 \%$ de los internos recibe visita de modo mensual mientras el $31.8 \%$ de los internos recibe visita de modo esporádico, según lo obtenido en la ficha sociodemográfica.

Con respecto a los niveles de depresión recogidos con el Inventario de Depresión de Beck. Los resultados se muestran en la tabla 3, en dónde el $28.7 \%$ de los internos tiene un nivel de depresión mínima, el $24.7 \%$ leve, el $22.4 \%$ moderada y el $24.2 \%$ grave. Llama la atención que el $24.2 \%$ de internos presenta depresión grave, ello puede explicarse por distintos factores como situación jurídica, inadaptabilidad al sistema penitenciario, problemas familiares, escasa visita, estar lejos 
de su familia, problemas con otros internos y problemas de encontrar un cupo de trabajo.

\section{Tabla 3}

Niveles de depresión en internos

\begin{tabular}{lcc}
\hline Niveles & $f$ & $\%$ \\
\hline Mínima & 109 & 28.7 \\
Leve & 94 & 24.7 \\
Moderada & 85 & 22.4 \\
Grave & 92 & 24.2 \\
\hline Total & 380 & 100.0 \\
\hline$n=380$ & &
\end{tabular}

En la tabla 4 se observa las prácticas religiosas más comunes que realizan los internos dentro del Establecimiento Penitenciario, las cuales son: rezar a Dios para pedirle ayuda (61.1\%), reza para agradecer a Dios $(56.1 \%)$, trasmite su fe a personas cercanas $(36.6 \%)$, lee la biblia $(25.5 \%)$ y asiste a las celebraciones religiosas que se realizan en el Establecimiento Penitenciario (25\%).

En la tabla 5, se observa la relación entre los indicadores de prácticas religiosas y la depresión, los resultados alcanzados al medir la relación de los indicadores de prácticas religiosas y depresión, en la prueba Chi-cuadrado al 95\% de confianza permiten concluir que; no existe relación entre los indicadores de la escala de prácticas religiosas y la depresión, se destaca que el indicador comportamiento idóneo guarda una relación negativa con la depresión, esto quiere decir, que las dos variables en este caso se correlacionan en un sentido inverso. 


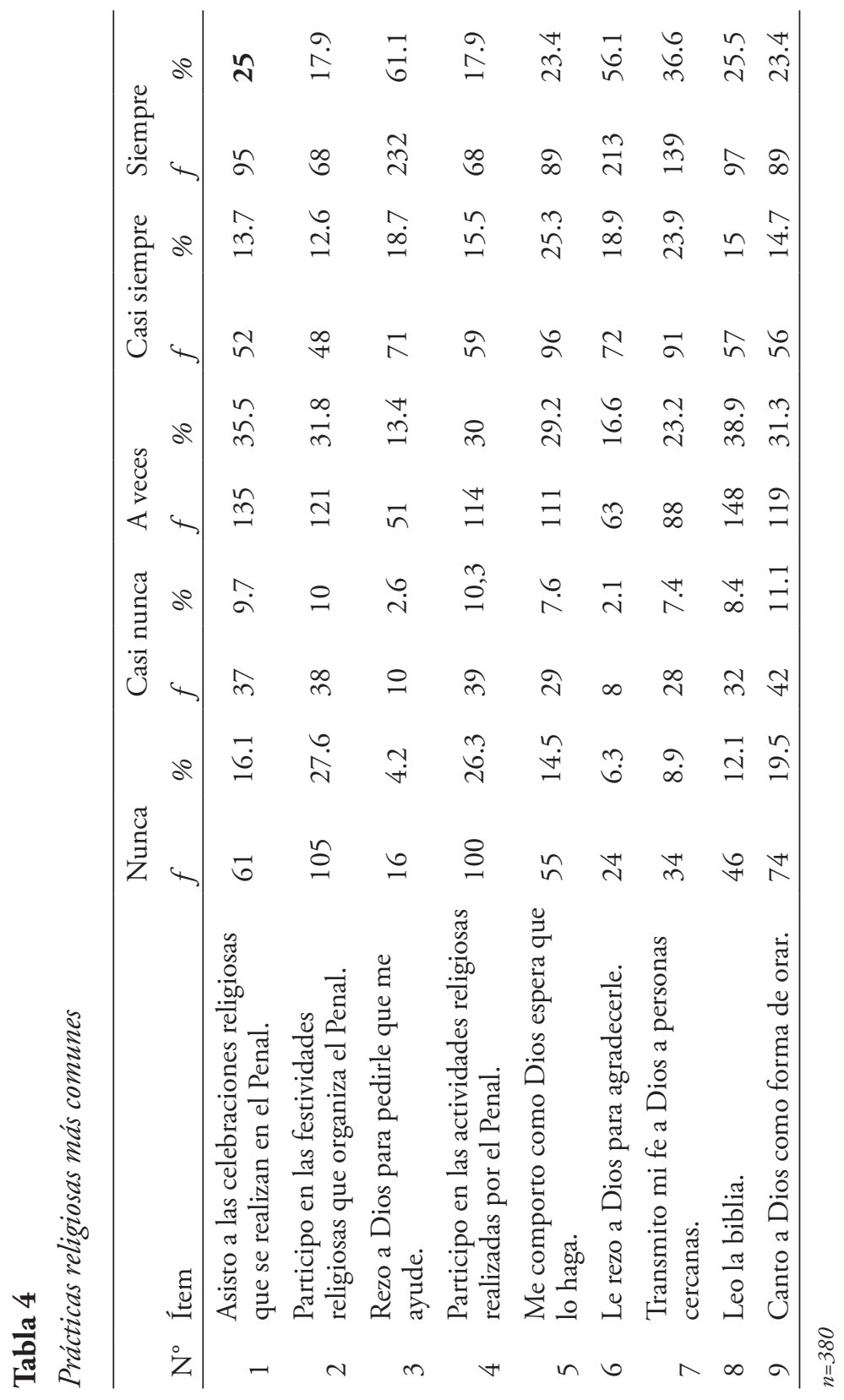




\section{Tabla 5}

Relación entre los indicadores de las prácticas religiosas con la depresión

\begin{tabular}{llccl}
\hline \multirow{2}{*}{$\mathrm{N}$} & Prácticas religiosas & \multicolumn{3}{c}{ Depresión } \\
\cline { 3 - 5 } & & $\begin{array}{c}\text { Tau }-\mathrm{b} \text { de } \\
\text { Kendall }\end{array}$ & $p$ & Relación \\
\hline 1 & Asistir a misa y/o culto & .016 & .727 & No existe \\
2 & Festividad religiosa & .048 & .261 & No existe \\
3 & Pedir ayuda a Dios & .014 & .757 & No existe \\
4 & Actividad religiosa & .006 & .166 & No existe \\
5 & Comportamiento idóneo & -.127 & .002 & Existe relación negativa \\
6 & Agradecer a Dios & .005 & .092 & No existe \\
7 & Transmitir la fe & -.01 & .814 & No existe \\
8 & Leer la biblia & -.052 & .242 & No existe \\
9 & Cantar y/o alabanza & -.042 & .325 & No existe \\
\hline
\end{tabular}

En la tabla 5, se observa que, de los 9 indicadores, el indicador comportamiento idóneo guarda relación negativa con la depresión de $-.127(p<.05)$.

\section{Discusión}

Según los resultados obtenidos, se encontró que no existe relación entre depresión y prácticas religiosas. Como se refiere en el cuerpo teórico, esto puede deberse a diversas razones, una de ellas sería que dichas prácticas podrían no estar motivados por la fe o que simplemente dichas prácticas no son adecuadas para la muestra de investigación (Viau, 2015). Otra sería la relación entre la religiosidad y la salud, donde la relación entre religiosidad y depresión es sumamente compleja puesto que distintas prácticas religiosas se relacionan de maneras distintas con este trastorno (Pérez et al., 2005). 
Este resultado va en la misma línea con los resultados hallados por Dasso (2010), quien no encontró correlación significativa entre ambas variables. No obstante, estos resultados difieren con Pérez et al. (2005) quienes encuentran una correlación negativa en un grupo religioso o espiritual, tanto en la muestra de hombres como de mujeres. También Gallardo y Sánchez (2014), muestran en sus resultados que solo la combinación de creencia y práctica religiosa se relaciona con menor incidencia de síntomas depresivos. Los resultados de estas dos últimas investigaciones difieren debido a que estas fueron realizadas en población no penitenciaria y en el extranjero. Esto es una prueba más de las controversias que hay en el estudio de la religiosidad y su posible relación con la salud mental en general.

Para explicar los hallazgos se retoma a Durkheim (como se citó en Rodríguez, 2015), ya que si bien los internos asumen prácticas religiosas, estas podrían estar obedeciendo más que a un factor de fe y actitud, a la obediencia mecánica del mandato social: hacer lo que se juzga que el colectivo espera de uno. Razón que cobra especial relevancia en quienes en su condición de internos buscan calificar con mejoras en su conducta para agilizar su libertad. Los internos que cumplen con las reglas y muestran buena conducta pueden reducir sus penas haciéndose merecedores a los beneficios penitenciarios.

Con respecto a los niveles de depresión se obtuvo como resultado que un $24.7 \%$ de los internos tienen depresión leve, el $22.4 \%$ depresión moderada, y el $24.2 \%$ depresión grave, por tanto, casi la cuarta parte de la muestra está en niveles de depresión moderada y grave. Este resultado se contrasta con los encontrados por el INEI (2016), así como con otras investigaciones regionales: Cárdeñas (2004) con su investigación Depresión y alcoholismo en un grupo de internos sentenciados en el Penal de Quenccoro - Cusco y Ccoya (2009) en su estudio Niveles de sintomatología depresiva en las internas del Establecimiento Penitenciario de Mujeres Cusco.

Según Mérida (2012), la depresión es un trastorno de estado de ánimo en el cual este se desplaza hacia la tristeza y la persona nota una considerable disminución de energía e interés en la mayoría de 
actividades de la vida diaria, que la limitan para desarrollarlas como lo hacía hasta ese momento, acompañado de un notable pesimismo que no corresponde con el carácter habitual de la persona. Estos cambios originan un deterioro del funcionamiento interpersonal y una pérdida de interés en los que le rodean. También disminuye el interés en la faceta laboral, puede dejar de ir a trabajar y supone en ocasiones la pérdida del puesto de trabajo. La persona puede abandonar hábitos de higiene y alimentación que previamente hacia sin ningún esfuerzo, y dejar de lado sus pasatiempos y actividades placenteras que tanto le gustaban hasta ese momento.

La depresión puede ir acompañada de síntomas físicos (disminución de peso o aumento, sudoración, otros). En resumen, la depresión es un trastorno afectivo del estado de ánimo que incapacita al que la sufre para disfrutar de las cosas de las que antes disfrutaba, caracterizada por sentimientos de inutilidad, culpa, tristeza, indefensión y desesperanza profundos. A diferencia de la tristeza normal, o la del duelo que sigue a la pérdida de un ser querido, la depresión patológica es una tristeza sin razón aparente que la justifique, y además, grave y persistente. Puede aparecer acompañada de varios síntomas parecidos, incluidas las perturbaciones del sueño y la comida, la pérdida de iniciativa, el autocastigo, el abandono, la inactividad y la incapacidad para el placer (Mérida, 2012).

Según el Ministerio de Sanidad Servicios Sociales e Igualdad de España (MSSSI, 2014), la depresión tiene una causa multifactorial, los que se pueden clasificar en factores personales, sociales, cognitivos, familiares y genéticos.

Para Beck et al., (2006), el contenido de los esquemas de depresión están constituidos por la triada cognitiva, que es la visión negativa de sí mismo, del mundo y del futuro. La modalidad depresiva presenta una serie de distorsiones sistemáticas en el procesamiento de la información, entre los que destaca: la sobre generalización, pensamiento dicotómico, inferencias arbitrarias, magnificación-minimización, abstracción selectiva y personalización. Estos sesgos sistemáticos en el procesamiento de la información, junto a la traída cognitiva producen las alteraciones en 
el procesamiento de la información que se manifiesta en la depresión. Por ende, el interno tiende a mantener su visión negativa de sí mismo, del mundo y del futuro, aunque pudiesen hacerse interpretaciones alternativas más plausibles. Las emociones y conductas típicas de la depresión están determinadas en gran medida por la forma en que la persona percibe la realidad y aún más, si esta se encuentra en contexto penitenciario.

Dadas las condiciones que se explicaron en la población de estudio, el $24.2 \%$ de internos presentan una depresión grave, porcentaje bajo a comparación de otras investigaciones como los de Cardeñas (2004) que muestra una prevalencia de depresión del 45.5\%. Ccoya (2009), obtuvo una prevalencia de $66.2 \%$ de depresión en la población estudiada. Valle (2017), concluyo que el índice de depresión en las internas es muy alto, debido a que el personal del área de tratamiento en los penales no se abastece. Estos niveles de depresión se puede deber a que hay insuficiente cobertura de las acciones de tratamiento.

Esta se fundamenta por el limitado equipamiento a nivel de educación y trabajo acorde con la realidad del interno, inexistencia de un sistema de tratamiento articulado entre las áreas de educación, trabajo, social, psicología y legal e insuficiente cobertura para la atención de salud integral, también por la existencia de niveles elevados de hacinamiento e infraestructura desfavorable para la rehabilitación del interno según el Ministerio de Justicia y Derechos Humanos (2015).

La mayoría de los estudios en población penitenciaria demuestran altos niveles de depresión en los grupos estudiados (Mojica et al., 2009; Medina et al., 2011; Moreno, 2012; Martínez, 2012). Solo León (2016) encontró un $90.16 \%$ de internos con un nivel de rango normal de depresión. Cabe resaltar que esta investigación fue realizada en internos por el delito de violación sexual con una muestra de 61 internos del Instituto Nacional Penitenciario de Lima, mientras que la muestra con la que se realizó el estudio fueron internos de distintos delitos pertenecientes a un Establecimiento Penitenciario de otra región del Perú.

Con respecto a las prácticas religiosas más comunes entre los internos del Establecimiento Penitenciario de Cusco se encontró: rezar 
a Dios para pedirle ayuda, trasmitir la fe de Dios a personas cercanas, leer la biblia y asistir a las celebraciones que se realizan dentro del Penal. Las prácticas religiosas son la relación entre Dios y uno mismo, lo cual es algo muy particular para cada persona y algo que debe ser muy autentico, íntimo y que se vive día a día. Lo más real, en este sentido, es lo que sirve para ser personas más amorosas, respetuosas, cariñosas, humildes, integras, puras y creativas (Vivanco, 2016).

Los investigadores del área de la psicología social, de la psicología de la religión y la espiritualidad destacan los beneficios y efectos positivos de las creencias, las prácticas y los rituales religiosos sobre la salud. En otras investigaciones se corrobora que la participación en redes sociales religiosas ayuda al afrontamiento de problemas y que a la vez disminuye el riesgo de estrés. Es así que se ha contrastado relaciones entre ciertos aspectos de religiosidad con la resolución o salida de situaciones traumáticas en los cuales la religión ha tenido una influencia positiva para la salud mental de las personas (Ellis, 1986).

La mayoría de los internos del Establecimiento Penitenciario de Cusco están afiliados a la religión católica. En el caso de estas personas sus prácticas religiosas son: seguir normas y ceremonias religiosas que puede significar un modo más institucionalizado de ser católico, mientras que hacer el bien a otras personas expresaría un sentimiento de ser católico al interior de un universo social más amplio que el de la propia iglesia y en el que se está en contacto e interacción con otros. Lo mismo podríamos decir de los evangélicos que ocupan el segundo lugar entre los internos, para quienes su condición es interpretada como compromiso con el prójimo, procurando ayudarlo a lograr la salvación (Sánchez, 2017).

Por otro lado, dentro del Establecimiento Penitenciario las prácticas religiosas son muy variadas según el tipo de religión, se encontró: la peregrinación o procesión, rezar en una capilla y concurrir a misas o cultos programadas por los movimientos religiosos en donde hacen sus alabanzas, rezos y lectura de la biblia. Otros estudios en población no penitenciaria cuentan con los mismos resultados (Martínez, 2014; López y Suarez, 2016; Vivanco, 2016). Se puede decir que las prácticas 
religiosas son más frecuentes en el ambiente penitenciario, cuando las personas atraviesan por situaciones difíciles, se interesan por conocer y vivir las creencias de la Iglesia Adventista (Vivanco, 2016). Asimismo, según Gallardo y Sánchez (2014), concluye que la pertenencia a una religión no constituye un factor de protección frente a los síntomas de depresión.

Finalmente, sobre la relación entre los indicadores de las prácticas religiosas y la depresión en los internos del Establecimiento Penitenciario, se encontró que solo un indicador de las prácticas religiosas muestra una relación inversa con la depresión: el indicador "Me comporto como Dios espera que lo haga". Esto implica que a un menor comportamiento como Dios espera, se tendrá un mayor nivel de depresión. El resultado obtenido contrasta con los resultados hallados por Dasso (2010), donde encontró una correlación de -.478 con el ítem 5 "Me comporto como Dios espera que lo haga". Según este indicador, los internos del Establecimiento Penitenciario se comportan como Dios espera que lo hagan en el entorno penitenciario, buscando primero un arrepentimiento por el delito cometido, y el daño generado a sus víctimas y familia. Segundo la obtención de beneficios penitenciarios modificando ciertas conductas negativas para tener un comportamiento adecuado, respetando la integridad física y psicológica, los principios éticos, morales y religiosos de los demás según el Reglamento del Código de Ejecución Penal (Decreto Supremo $\mathrm{N}^{\circ} 15$, 2003).

\section{Conclusiones}

Se concluye que no existe relación entre depresión y las prácticas religiosas en internos del Establecimiento Penitenciario. Asimismo, se obtuvo que el $71 \%$ de la muestra investigada tiene algún nivel de depresión. De estos, el 24.2\% tiene depresión grave, el $22.4 \%$ de los internos tiene depresión moderada y $24.7 \%$ depresión leve. 
Por otra parte, las prácticas religiosas más comunes realizadas por internos dentro del Penal de Cusco son; 61.1\% rezar a Dios para pedir ayuda, $56.1 \%$ agradece a Dios, $36.6 \%$ trasmite su fe a personas cercanas, $25.5 \%$ lee la biblia y $25 \%$ asiste a celebraciones religiosas que se realizan dentro del Establecimiento Penitenciario.

Con respecto a la relación de los indicadores de la escala de prácticas religiosas con la depresión se concluye que el indicador de "Me comporto como Dios espera que lo haga", guarda una relación significativa con la depresión. Es decir, a menor comportamiento como Dios espera que lo haga, mayor depresión. Con respecto al indicador "Me comporto como Dios espera que lo haga", este ayuda al interno en su buen comportamiento dentro del Establecimiento Penitenciario para poder obtener beneficios dentro de este ambiente, así como para no ser castigados por el personal del INPE y compañeros de cuadra.

\section{Referencias}

Acosta, R., y Sánchez, J. (2017). Religiosidad y depresión en adultos mayores institucionalizados de Lima Metropolitana. (Tesis de licenciatura). Universidad Peruana de Ciencias Aplicadas (UPC). Lima, Perú.

Asociación Psiquiátrica Peruana. (2011). Seis de cada 10 reos son psicóticos. Perú21. Recuperado de https://peru21.pe/lima/ seis-10-reos-son-psicoticos-7247.

Beck, A., Steer, R., y Brown, G. (2006). Inventario de depresión de Beck (BDI-II). Buenos Aires: Paidós.

Berger, L. (1974). La construcción social de la realidad. Trad. Silvia Zuleta. Amorrortu, Buenos Aires.

Borja, C., Aguado, T., y Urquizo, J. (2006). La mujer en el sistema penitenciario peruano. Lima y Junta de Andalucía: IDEMSA.

Castro, N. (2009). Realidad penitenciaria y derechos humanos: Penal de Lurigancho (Perú). (Tesis de maestría). Universidad Internacional de Andalucía. Lima, Perú. 
Cardeñas, Z. (2004). Depresión y alcoholismo en un grupo de internos sentenciados en el Establecimiento Penal Quenccoro - Cusco. (Tesis de maestría). Universidad Cayetano Heredia. Lima, Perú.

Ccoya, W. (2009). Niveles de sintomatología depresiva en las internas del Establecimiento Penitenciario de Mujeres del Cusco. (Tesis de licenciatura). Universidad Andina del Cusco, Perú.

Chaves, É., Paulino, C., Souza, V., Mesquita, A. C., Carvalho, F., y Nogueira, D. (2014). Qualidade de vida, sintomas depressivos e religiosidade em idosos: um estudo transversal. Texto Contexto Enferm 23(3). 648-655.

Centro Nacional de Estudios Criminológicos y Penitenciarios. (2007). Diagnóstico de Salud Mental en Establecimientos Penitenciarios. Lima: CENECP.

Dasso, A. (2010). Sintomatología depresiva y prácticas religiosas en internas por delitos comunes de un penal de Lima. (Tesis de licenciatura). Pontifica Universidad Católica del Perú. Lima, Perú.

Decreto Supremo No 015-2003-JUS. (2003). Reglamento del Código de Ejecución Penal. Recuperado de http://www2.congreso.gob.pe/ sicr/cendocbib/con4_uibd.nsf/D04A8DAD08FE381A05257B F8008222BA/\$FILE/18.pdf

Defensoría del Pueblo (2011). Decimoquinto Informe Anual de la Defensoría del Pueblo. Recuperado de https://www.defensoria.gob.pe/ wp-content/uploads/2018/05/15-informe-anual-dp.pdf

Defensoría del Pueblo (2017). Vigésimo Primer Informe Anual. Recuperado de https://www.defensoria.gob.pe/wp-content/ uploads/2018/07/Informe_anual_DP_2017.pdf

Ellis, A. (1986). El caso contra la religión: A visión del psicoterapeuta y el caso en contra religiosidad. Austin Tx: American Ateist Press.

Gallardo, L., y Sánchez, E. (2014). Participación religiosa y depresión en personas mayores de la región de Arica y Parinacota, Chile.

Hamann, G. (2003). Religión y actitudes hacia el tratamiento en pacientes esquizofrénico. (Tesis de licenciatura no publicada). Pontificia Universidad Católica del Perú. Lima, Perú. 
Instituto Nacional de Estadística e Informática. (2016). Primer Censo Nacional Penitenciario. Perú.

Instituto Nacional Penitenciario. (2011). Manual de Normas de Tratamiento Penitenciario - INPE.

Instituto Nacional Penitenciario. (2017). Informe estadistico setiembre 2017. Lima, Perú.

Instituto Nacional Penitenciario. (2018). Funcionarios penitenciarios unifican criterios con agentes pastorales. Nota de prensa $N^{\circ}$ 075 -2018- INPE. Recuperado de https://www.inpe.gob.pe/ noticias-region-norte/item/1508-funcionarios-penitenciariosunifican-criterios-con-agentes-pastorales.html.

León, A. (2016). Clima social familiar y la depresión de los internos por delito de violación del Instituto Nacional Penitenciario Lima. (Tesis de licenciatura). Universidad Católica Los Ángeles de Chimbote, Perú.

Ley $\mathrm{N}^{\circ}$ 15. Diario Oficial del Bicentenario El Peruano, Lima, Perú, 2003.

López, J., y Suarez, A. (2016). Diversidad de creencias, devociones y prácticas religiosas en los asentamientos precarios de la Ciudad de Buenos Aires. Buenos Aires. https://doi.org/10.1590/0100-8587 2016v36n1cap05

Martínez, C. (2012). Depresión en hombres reclusos de la zona sur del Estado de Veracruz. Tesis de licenciatura, Universidad Veracruzana, Veracruz, México. Recuperado de http://cdigital.uv.mx/ bitstream/123456789/31635/1/martinezmartinezciro.pdf

Martínez, M. (2014). Religiosidad, Prácticas Religiosas y Bienestar Subjetivo en Jóvenes Católicos de Lima Norte. (Tesis de licenciatura). Pontifica Universidad Católica del Perú. Lima, Perú.

Medina, O., Cardona, D., y Arcila, S. (2011). Riesgo suicida y Depresión en un grupo de internos de una cárcel de Quindio (Colombia). Fundación Universitaria del Área Andina. Bogotá, Colombia.

Mendoza, R. (2013, Marzo 30). Depresión y estrés tras las rejas. La Republica. Recuperado de https://larepublica.pe/ archivo/700828-depresion-y-estres-tras-las-rejas. 
Mérida, J. (2012). Nivel de depresión en personas privadas de libertad" (estudio realizado en la cárcel de la Comisaría 42, Policia Nacional Civil, San Marcos, S.M. (Tesis de licenciatura). Universidad Rafael Landívar. Quetzaltenango, México.

Mikulic, I., \& Crespi, M. (2005). Contexto carcelario: un estudio de los estresores y las respuestas de afrontamiento en detenidos primarios y reincidentes. Anuario de Investigaciones - Facultad de Psicología, Universidad de Buenos Aires, 12, 211- 218. Recuperado de http://www.scielo.org.ar/scielo.php?script=sci_arttext\&pid= S1851-16862005000100021\&lng=es\&nrm=iso>.

Ministerio de Justicia y Derechos Humanos. (2015). Infraestructura penitenciaria: proyección de la capacidad de albergue del 2015 al 2035.

Ministerio de Sanidad, Servicios Sociales e Igualdad. (2014). Guía de Práctica Clinica sobre el Manejo de la Depresión en el Adulto. España. Recuperado de http://www.guiasalud.es/GPC/ GPC_534_Depresion_Adulto_Avaliat_compl.pdf

Mojica, C., Sáenz, D. y Rey-Anacona, C. (2009). Relación entre variables asociadas al encarcelamiento y sentimientos de desesperanza, depresión y riesgo suicida en internos del Establecimiento Carcelario El Olivo de Santa Rosa de Viterbo. (Tesis de licenciatura). Universidad Pedagógica y Tecnológica de Colombia, Tunja, Colombia. Moreno, E. (2012). La depresión y sus efectos en la capacidad mental de las personas privadas de libertad (PPL). (Tesis de licenciatura). Universidad Central del Ecuador. Quito, Ecuador.

Mori, N., y Caballero, J. (2010). Historia natural de la depresión. Revista Peruana de Epidemiología, 14 (2), 86-90.

Ocampo, J., Romero, N., Herrera, J., y Reyes, C. (2006). Prevalencia de las prácticas religiosas, disfunción familiar, soporte social y síntomas depresivos en adultos mayores. Colombia Médica, $37(2), 26-30$.

Ordóńez, L., y Ruiz, J. (2008). Suicidio en prisión. Recuperado de http://extension.upbbga.edu.co/inpec2009/Estudiosprimeraparte/areasdisciplinares/psicologia.pdf. 
Organización Mundial de la Salud. (2017). Décima Revisión de la Clasificación Internacional de Enfermedades. Madrid: Meditor.

Palmer, E y Connelly, R. (2005). Depresión, desesperanza en ideación suicidio entre presos vulnerables. CBMH Conducta criminal y salud mental. Recuperado de https://onlinelibrary.wiley.com/ doi/pdf/10.1002/cbm.4\#citedby-section.

Penal Reform International. (2015). Global prisión trends. Recuperado de htttp://www. Penaireform.org/wp-content/uploads/2015/04/ PRI-Prisions-global-trens-report-LR-pdf.

Pérez, A., Sandino, C., y Gómez, V. (2005). Relación entre depresión y práctica religiosa: estudio exploratorio. (Tesis de licenciatura). Universidad de los Andes, Colombia.

Rodríguez, J. (2015, Mayo 2015). Emile Durkheimyloelemental dela vida religiosa. 80 grados sin prisa. Recuperado de http://www.80grados. net/emile-durkheim-y-lo-elemental-de-la-vida-religiosa/

Ruiz, J. (2007). Síntomas psicológicos, clima emocional, cultura y factores psicosociales en el medio penitenciario. Revista Latinoamericana de Psicología, 39, 547-561.

Sánchez, J. (2017). Religiones y religiosidad en el Perú de hoy. Boletín $\mathrm{N}^{\circ} 148$. Estado de la Opinión Pública. Lima, Perú.

Valverde, J. (1991). La cárcel y sus consecuencias: La intervención sobre la conducta desadaptada. Madrid: Popular.

Valle, B. (2017). Depresión en Internas del pabellón A, B y C del Penal de Mujeres de Chorrillos - Lima. (Tesis de licenciatura). Universidad Inca Garcilaso de la Vega. Lima, Perú.

Vexler, I. (2003). Informe sobre la educación peruana: Situación y perspectivas. Recuperado de file://C:/Users/Alejandrino/Downloads/ ibeperu.pdf

Vivanco, L. (2016). Práctica religiosa y adhesión al estilo de vida adventista de los jóvenes de la misión del oriente peruano en el 2015. (Tesis de maestría). Universidad Peruana Unión. Lima, Perú.

Recibido: 29/05/2019

Revisado: 06/10/2020

Aceptado: 08/10/2020 